\title{
Free yang-mills theory versus toric Sasaki-Einstein manifolds
}

AUTHOR(S):

Nishioka, T; Takayanagi, T

CITATION:

Nishioka, T ...[et al]. Free yang-mills theory versus toric Sasaki-Einstein manifolds. PHYSICAL REVIEW D 2007, 76(4): 044004.

ISSUE DATE:

2007-08

URL:

http://hdl.handle.net/2433/49941

RIGHT:

Copyright 2007 American Physical Society 
PHYSICAL REVIEW D 76, 044004 (2007)

\title{
Free Yang-Mills theory versus toric Sasaki-Einstein manifolds
}

\author{
Tatsuma Nishioka* and Tadashi Takayanagi ${ }^{\dagger}$ \\ Department of Physics, Kyoto University, Kyoto 606-8502, Japan \\ (Received 16 May 2007; published 8 August 2007)
}

\begin{abstract}
It has been known that the Bekenstein-Hawking entropy of the black hole in $\operatorname{AdS}_{5} \times S^{5}$ agrees with the free $\mathcal{N}=4$ super Yang-Mills entropy up to the famous factor $\frac{4}{3}$. This factor can be interpreted as the ratio of the entropy of the free Yang-Mills theory to the entropy of the strongly coupled Yang-Mills theory. In this paper we compute an analogous factor for infinitely many $\mathcal{N}=1$ superconformal field theories (SCFTs) which are dual to toric Sasaki-Einstein manifolds. We observed that this ratio always takes values within a narrow range around $\frac{4}{3}$. We also present explicit values of volumes and central charges for new classes of toric Sasaki-Einstein manifolds.
\end{abstract}

DOI: 10.1103/PhysRevD.76.044004

PACS numbers: 04.70.Dy, 04.50.+h

\section{INTRODUCTION}

The anti-de Sitter/conformal field theory (AdS/CFT) correspondence [1] has been playing a crucial role in exploring the nonperturbative aspects of gravity and gauge theories for ten years. Even though there are many important examples of AdS/CFT, a general condition that a given CFT should have its AdS dual has not been known completely until now. One simple strategy to understand this issue better is to study many examples of AdS/CFT systematically and see if there are any common properties for CFTs which have their AdS duals.

Fortunately, infinitely many $\mathcal{N}=1$ superconformal field theories (SCFTs) have recently been known to have their AdS duals in terms of the five-dimensional SasakiEinstein manifolds $X_{5}[2-4]$, generalizing the celebrated example $T^{1,1}$ [5]. Therefore it is very interesting to find any common properties among them. A basic and physically important quantity will be the degrees of freedom of a given CFT. We can estimate this by computing its thermodynamical entropy at finite temperature. ${ }^{1}$

We can easily compute the entropy of a super YangMills theory in its strong coupling limit as the entropy of the dual black hole $[7,8]$. On the other hand, it is very difficult to calculate the entropy directly in the strongly coupled Yang-Mills theory. Instead we assume a free YangMills approximation of SCFTs. This crude approximation works better than we naively expect, due to the super conformal symmetry. Indeed it has been known that this approximation deviates from the dual gravity result only by the factor $\frac{4}{3}$ in the $\mathcal{N}=4$ super Yang-Mills theory [7]. Since this semiquantitative agreement is a remarkable property, it is intriguing to see if a similar agreement is true for other SCFTs which have their AdS duals.

\footnotetext{
*nishioka@gauge.scphys.kyoto-u.ac.jp

†takayana@gauge.scphys.kyoto-u.ac.jp

${ }^{1}$ Another way to measure the degrees of freedom will be to count the Bogomol'nyi-Prasad-Sommerfield (BPS) states of a given SCFT. This has been discussed in [6], recently.
}

Furthermore, it has been pointed out that even the Hagedorn transition in the dual string theory on $\mathrm{AdS}_{5}$ can also be captured from the free Yang-Mills theory [9].

The aim of this paper is to investigate this ratio $\frac{S_{\text {free }}}{S_{\text {strong }}}$ of the entropy of various $\mathcal{N}=1$ quiver gauge theories with all interactions turned off, to the entropy in the strongly coupled $\mathcal{N}=1$ SCFTs realized as IR fixed points of the interacting quiver theories. We can physically interpret this ratio as a measure of the strength of interactions in a given CFT. We can compute the black hole entropy, which is inversely proportional to the volume of Sasaki-Einstein manifolds, by employing the $Z$-minimization method [10] dual to the $a$-maximization [11-14]. Therefore we can obtain this ratio only from the information of the toric data for any toric Sasaki-Einstein manifolds. After we search large families of infinitely many toric diagrams, we find that the ratio is always in a narrow range $\frac{8}{9} \leq \frac{3}{4}$. $\frac{S_{\text {free }}}{S_{\text {strong }}} \lessgtr 1.2$. The minimum value $\frac{S_{\text {free }}}{S_{\text {strong }}}=\frac{32}{27}$ is realized when $X_{5}$ is equal to $T^{1,1}$ or its orbifolds.

This paper is organized as follows. In Sec. II we give the expression of the ratio $\frac{S_{\text {free }}}{S_{\text {strong }}}$ in terms of the volume of Sasaki-Einstein manifolds and the number of fields in the dual gauge theory. In Sec. III we calculate this ratio explicitly for various examples. In Sec. IV we compute an analogous ratio in $\mathcal{N}=1 \mathrm{SQCD}$. In Sec. V, we summarize our results and also discuss other interesting quantities.

\section{ENTROPY FROM BLACK HOLE AND FREE YANG-MILLS THEORY}

Consider a background $\mathrm{AdS}_{5} \times X_{5}$ in type IIB supergravity, where $X_{5}$ is a five-dimensional Sasaki-Einstein manifold. This theory is dual to a four-dimensional $\mathcal{N}=$ 1 SCFT [5]. Such a theory is explicitly described by a $S U(N)$ quiver gauge theory [3,5]. A systematic construction of such gauge theories is recently found by using the brane-tiling method $[15,16]$ (for recent progresses see e.g. [17-20]). 
The AdS radius is found to be

$$
R=\left(\frac{4 \pi^{4} g_{s} \alpha^{2} N}{\operatorname{Vol}\left(X_{5}\right)}\right)^{1 / 4}
$$

where $\operatorname{Vol}\left(X_{5}\right)$ is the volume of $X_{5}$ normalized such that $\operatorname{Vol}\left(S^{5}\right)=\pi^{3}$ [21]. The central charge $a$ of the SCFT [22] is related to the volume via

$$
a=\frac{N^{2}}{4} \cdot \frac{\pi^{3}}{\operatorname{Vol}\left(X_{5}\right)} .
$$

The thermodynamical entropy $S$ in the strong coupling limit of the SCFT can be found from the entropy of the AdS-Schwarzschild black hole ${ }^{2}(T$ is the temperature)

$$
S_{\text {strong }}=\frac{\text { horizon area }}{4 G_{N}^{(5)}}=\frac{\pi^{5} N^{2}}{2 \operatorname{Vol}\left(X_{5}\right)} V T^{3}=2 \pi^{2} a V T^{3},
$$

where $G_{N}^{(5)}$ is the $5 \mathrm{D}$ Newton constant and it is related to the 10D Newton constant $G_{N}^{(10)}=8 \pi^{6} \alpha^{14} g_{s}^{2}$ via the dimensional reduction $G_{N}^{(10)}=R^{5} \operatorname{Vol}\left(X_{5}\right) \cdot G_{N}^{(5)}$. The entropy is proportional to the central charge and this is consistent with the expectation that $a$ is related to the degrees of freedom.

However, we would like to notice that the entropy is not always proportional to $a$ for all coupling regions. For example, in the free super Yang-Mills theory, the entropy is proportional to the number of bosons $N_{B}$ as the contribution of a (free) gauge field $A^{\mu}$ is the same as a (free) complex scalar field $\Phi$ (we count each of these as a unit $N_{B}=1$.). Since the central charge $a$ of $A^{\mu}$ is different from that of $\Phi$, the free Yang-Mills entropy is not proportional to a.

Therefore it is interesting to consider the ratio $\frac{S_{\text {free }}}{S_{\text {strong }}}$ of the free Yang-Mills entropy to the strongly coupled YangMills entropy. This ratio measures how the degrees of freedom change when we turn on the interactions of the quiver gauge theories. It is well known that $\frac{S_{\text {free }}}{S_{\text {strong }}}$ becomes $\frac{4}{3}$ for the $\mathcal{N}=4$ super Yang-Mills theory [7]. In general $\mathcal{N}=1$ SCFTs, we have to worry about the ambiguity of the field content of the free field approximation due to the Seiberg duality. ${ }^{3}$ Even though the entropy in the free YangMills theory depends on the frame of Seiberg duality or equally on the choice of toric phases of $X_{5}$ [23], we will find that this ambiguity changes the entropy only slightly in explicit examples. Thus this does not spoil our semiquantitative argument in this paper. We will proceed the arguments by choosing a standard toric phase.

In the end, this ratio can be computed as follows via AdS/CFT

\footnotetext{
${ }^{2}$ In this paper we consider the AdS black holes in the Poincare coordinate.

${ }^{3}$ We are very grateful to Yuji Tachikawa for pointing out this issue to us in detail.
}

$$
\frac{S_{\text {free }}}{S_{\text {strong }}}=\frac{4}{3} \cdot \frac{N_{B}}{4 N^{2}} \cdot \frac{\operatorname{Vol}\left(X_{5}\right)}{\pi^{3}}=\frac{4}{3} \cdot \frac{N_{B}}{16 a} \equiv \frac{4}{3} f,
$$

where we defined the ratio $f$ normalized such that $f=1$ for the $\mathcal{N}=4$ super Yang-Mills theory. We will present results below in terms of this ratio $f$. A larger value of $f$ means that the degrees of freedom are more reduced in the strongly coupled regime compared with the free YangMills theory. As we will see later, $f$ takes values of order 1 (i.e. $\sim(1 \pm 0.2))$ in all examples we studied. In the orbifold theories, we always find $f=1$. Moreover, the value of $f$ remains the same after we take a $Z_{n}$ orbifold of any Sasaki-Einstein manifold $X_{5}$. Notice that the confirmation that the ratio is always of order 1 is already a nontrivial check of the AdS/CFT duality for infinitely many SCFTs.

We can represent other physical quantities in terms of $f$. The analogous ratio $\frac{E_{\text {free }}}{E_{\text {strong }}}$ of the Casimir energy in a super Yang-Mills theory compactified on a thermal circle [24] is the same as before $\frac{E_{\text {free }}}{E_{\text {strong }}}=\frac{4}{3} f$. Also the ratio of entanglement entropy [25] becomes $\frac{S_{A}^{\text {free }}}{S_{A}^{\text {strong }}}=\frac{2}{3} f$ [26] when we define the subsystem $A$ by dividing the boundary into two halfplanes.

We would like to stress again that the ratio $f$ is essentially (the inverse of) the central charge $a$ divided by the number of free fields $N_{B}$. The central charge itself increases (linearly) as the size of the toric diagram grows ${ }^{4}$ [27]. Since the number of fields $N_{B}$ also scales linearly as the area of the diagram becomes infinitely large, the ratio $f$ stays finite.

\section{ENTROPY FROM TORIC SASAKI-EINSTEIN MANIFOLDS}

A classification of the toric Sasaki-Einstein manifolds can be obtained by using the toric diagrams $[10,28]$ which describe corresponding Calabi-Yau cones. Though originally the toric diagrams are three dimensional for the cones over $X_{5}$, we can project them onto a two-dimensional plane due to the Calabi-Yau condition. Thus we can write the coordinates of vertices in the toric diagrams with $n$ vertices (i.e. $n$ polygon) as $\left(1, p_{i}, q_{i}\right) \in \mathbf{Z}^{3}(i=1,2, \cdots, n)$. We can compute several physical quantities of a quiver gauge theory from the toric diagram of its dual Sasaki-Einstein manifold.

The number of vector multiplets and chiral multiplets in a standard choice of the toric phase is given by [3]

$$
\begin{aligned}
& N_{\text {gauge }}=2 \cdot(\text { area of the toric diagram }), \\
& N_{\text {matter }}=\sum_{1 \leq i<j \leq n}\left|p_{i} q_{j}-p_{j} q_{i}\right| .
\end{aligned}
$$

\footnotetext{
${ }^{4}$ This can also be seen in explicit examples. It is clear from the result (3.2) for $Y^{p, q}$ that the corresponding central charge behaves as $a \propto p$ in the limit $p \rightarrow \infty$ with $p / q$ kept finite.
} 
FREE YANG-MILLS THEORY VERSUS TORIC SASAKI- . .

TABLE I. Table of the values of $f$ for various Sasaki-Einstein manifolds considered in Sec. III. We gave the maximum value $f_{\max }$ of $f$ as the minimum value is always $\frac{8}{9}$.

\begin{tabular}{|c|c|c|}
\hline SE manifolds & $f_{\text {max }} \quad N_{\text {gauge }} / N^{2}$ & $N_{\text {matter }} / N^{2}$ \\
\hline$Y^{p, q}$ & $1.02459 \quad 2 p$ & $4 p+2 q$ \\
\hline$L^{p, q, r}$ & $1.02459 p+q$ & $p+3 q$ \\
\hline$X^{p, q}$ & $1.037292 p+1$ & $4 p+2 q+1$ \\
\hline$Z^{p, q}$ & $1.050072 p+2$ & $4 p+2 q+2$ \\
\hline & $1.031722 p(2 r-q)$ & $2 p(4 r-q)$ \\
\hline \multicolumn{3}{|c|}{ (symmetric pentagon) } \\
\hline \multicolumn{3}{|c|}{ (symmetric hexagon) } \\
\hline Regular polygo & $1.09662 n \sin \left(\frac{2 \pi}{n}\right)$ & $2 n \sin \left(\frac{2 \pi}{n}\right)(n:$ even $) 2 n \sin \left(\frac{\pi}{n}\right)\left(1+\cos \left(\frac{\pi}{n}\right)\right)(n:$ odd \\
\hline
\end{tabular}

The above formula shows that $N_{\text {gauge }}$ is the same as the Euler number of the toric manifold. This is because the Euler number counts the number of independent (fractional) D branes. On the other hand, $N_{\text {matter }}$ is found by considering intersections of 3-cycles in the mirror CalabiYau cone in [3].

Using this, the number of total bosons is found to be $N_{B}=N_{\text {gauge }}+N_{\text {matter }}$. Notice that in the above formula (3.1) of the number of matter fields $N_{\text {matter }}$, we are choosing a particular toric phase. We will be able to employ other equivalent descriptions of the quiver gauge theories by applying the Seiberg's duality where the number of matter fields $N_{\text {matter }}$ takes different values [23]. As we will see below in the explicit example of $Y^{p, q}$, this ambiguity changes the value of the ratio $f$ only slightly, though we cannot give a complete general discussion on this issue since its systematic treatment has not been developed well at the present. Thus we will compute the ratio $f$ by choosing a particular choice of the toric phase by using the formula (3.1) in most of the examples in this paper. We argue that the ambiguity of the toric phases does not spoil our semiquantitative arguments in this paper because it does not affect the ratio $f$ substantially as mentioned. Also notice that in spite of this subtle ambiguity of the physical definition of the ratio $f$, this quantity is completely well defined after we plug (3.1) into the formula. This means that this value is mathematically exactly well defined as we can find a unique value when a toric diagram is given. Thus this quantity is also very interesting from this mathematical viewpoint.

Quite recently, the existence and uniqueness of the Sasaki-Einstein metric have been proved in [28] if a given toric diagram satisfies a simple condition (such a toric diagram is called good). ${ }^{5}$ First we will study all toric diagrams described by four vertices and then we examine

\footnotetext{
${ }^{5}$ This condition essentially requires that $p_{i}-p_{i+1}$ and $q_{i}-$ $q_{i+1}$ are coprime for all $i$ (refer to the second paper of [28]).
}

some particular classes with five or more vertices. To summarize the results obtained in this section, we will draw up the Tables I and II.

\section{A. Toric diagrams with four vertices}

\section{1. $Y^{p, q}$}

As a first example, we consider the familiar example $Y^{p, q}$ [2] whose toric diagram is given by Fig. 1 ( $p$ and $q$ are integers such that $p \geq q \geq 0$ ). The volume of $Y^{p, q}$ is known to be

$$
\frac{\operatorname{Vol}\left(Y^{p, q}\right)}{\pi^{3}}=\frac{q^{2}\left(2 p+\sqrt{4 p^{2}-3 q^{2}}\right)}{3 p^{2}\left(3 q^{2}-2 p^{2}+p \sqrt{4 p^{2}-3 q^{2}}\right)} .
$$

The number of bosons which appear in the dual field theory [3] is given by $\frac{N_{B}}{N^{2}}=2(3 p+q)$, where we have employed the explicit values (3.1) in the standard choice of toric phase. As a result, the ratio $f$ is expressed as follows

$$
f(x)=\frac{x^{2}(3+x)\left(2+\sqrt{4-3 x^{2}}\right)}{6\left(3 x^{2}-2+\sqrt{4-3 x^{2}}\right)}, \quad x \equiv \frac{q}{p} .
$$

This is plotted in Fig. 2 and the function $f(x)(0 \leq x \leq 1)$ takes the values within the range

$$
\frac{8}{9} \leq f(x) \leq 1.02459 \text {. }
$$

Notice that at $x=0$, where $X_{5}$ becomes the orbifold $T^{1,1} / Z_{p}$, the function $f(x)$ takes its minimum value $f=$ $\frac{8}{9}$. The maximum value in (3.4) is attained when $x=$ 0.76929 . On the other hand, at $x=1$, where $X_{5}$ is the orbifold $S^{5} / Z_{2 p}$, the function $f(x)$ takes $f=1$.

Now, let us ask how the ratio $f$ depends on the choice of toric phases. All toric phases in $Y^{p, q}$ have been obtained in [23]. The number of matter fields takes the following range

$$
6 p+2 q \leq \frac{N_{B}}{N^{2}} \leq 8 p,
$$



TABLE II. Table of the values of $f$ and the volumes for various Sasaki-Einstein manifolds
defined by the specified four-dimensional surfaces in the corresponding Calabi-Yau cones. The final example is defined by the octagonal toric diagram whose vertices are given by $(1,0),(2,0)$, $(3,1),(3,2),(2,3),(1,3),(0,2)$, and $(0,1)$ after being projected to the two-dimensional plane $R^{2}$.

\begin{tabular}{lcccc}
\hline \hline SE manifolds & $f$ & $\operatorname{Vol}\left(X_{5}\right) / \pi^{3}$ & $N_{\text {gauge }} / N^{2}$ & $N_{\text {matter }} / N^{2}$ \\
\hline$d P_{0}=\mathbb{P}^{2}\left(S^{5}\right)$ & 1 & $\frac{1}{3}$ & 3 & 9 \\
$d P_{1}\left(Y^{2,1}\right)$ & $\frac{322+91 \sqrt{13}}{648}=1.00325$ & $\frac{46+13 \sqrt{13}}{324}$ & 4 & 10 \\
$d P_{2}\left(X^{2,1}\right)$ & $\frac{118+22 \sqrt{33}}{243}=1.00568$ & $\frac{59+11 \sqrt{33}}{486}$ & 5 & 11 \\
$d P_{3}\left(Z^{2,1}\right)$ & 1 & $\frac{2}{9}$ & 6 & 12 \\
$\mathrm{SP}\left(X^{1,1}\right)$ & $\frac{5 \sqrt{3}}{9}=0.96225$ & $\frac{2 \sqrt{3}}{9}$ & 3 & 7 \\
$P d P_{4}$ (toric) & 0.96964 & 0.17630 & 7 & 15 \\
$d P_{4}$ (nontoric) & $\frac{55}{54}=1.01852$ & $\frac{5}{27}$ & 7 & 15 \\
& $\frac{28}{27}=1.03704$ & $\frac{8}{81}$ & 14 & 28 \\
(symmetric octagon) & & & & \\
\hline \hline
\end{tabular}

if we search all toric phases. The lowest value is the one (3.1) we employed in the above and this leads to the result (3.4). On the other hand, if we compute the ratio $f$ by using the highest possible value $\frac{N_{B}}{N^{2}}=8 p$, then the ratio takes the following range

$$
1<f_{\text {high }}<\frac{32}{27}
$$

This deviates from (3.4) less than 20\%. Thus we expect that our particular choice (3.1) of the toric phase does not spoil our semiquantitative arguments in this paper.

\section{2. $L^{p, q, r}$}

We move onto the toric Sasaki-Einstein manifolds $L^{p, q, r}$. This class includes all examples whose toric diagrams consist of four vertices as shown in Fig. 3. Define $x \equiv \frac{p}{q}$, $y \equiv \frac{r}{q}$ and then they should satisfy $0 \leq x \leq y \leq 1, y \leq$ $\frac{x+1}{2}$. The relation between $Y^{p, q}$ and $L^{p, q, r}$ is given by $Y^{p, q}=L^{p-q, p+q, p}$ and thus $Y^{p, q}$ is on the line $y=\frac{x+1}{2}$. The volume of $L^{p, q, r}$ is found to be as follows [4]

$$
\operatorname{Vol}\left(L^{p, q, r}\right)=\pi^{3} \cdot \frac{(p+q)^{3} W}{8 p q r s},
$$

where $W$ is the solution to the quartic equation

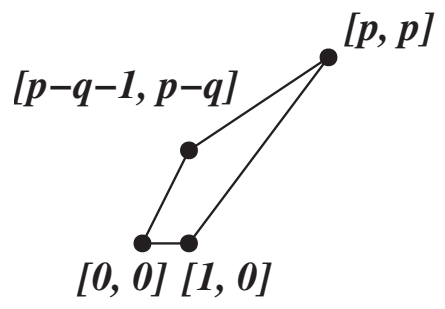

FIG. 1. Toric diagram of $Y^{p, q}$.

$$
\begin{array}{r}
\left(1-F^{2}\right)\left(1-G^{2}\right) h_{-}^{4}+2 h_{-}^{2}\left[2\left(2-h_{+}\right)^{2}-3 h_{-}^{2}\right] W \\
+\left[8 h_{+}\left(2-h_{+}\right)^{2}-h_{-}^{2}\left(30+9 h_{+}\right)\right] W^{2} \\
+8\left(2-9 h_{+}\right) W^{3}-27 W^{4}=0 .
\end{array}
$$

Here we defined $F=\frac{1-x}{1+x}, G=\frac{2 y-x-1}{1+x}, h_{ \pm}=F^{2} \pm G^{2}$. There are four solutions to this equation and only one of them is a positive real number. We use this solution for computing the volume of $L^{p, q, r}$.

The number of bosons in the dual gauge theory $[16,29]$ is given by $\frac{N_{B}}{N^{2}}=2(p+2 q)$. In the end, the ratio $f(x, y)$ is expressed as follows

$$
\begin{gathered}
f(x, y)=\frac{(1+x)^{3}(2+x) W(x, y)}{x y(1+x-y)}, \\
\left(x \leq y, y \leq \frac{x+1}{2}\right) .
\end{gathered}
$$

The numerical analysis shows that the range of $f(x, y)$ is the same as the one for $Y^{p, q}$

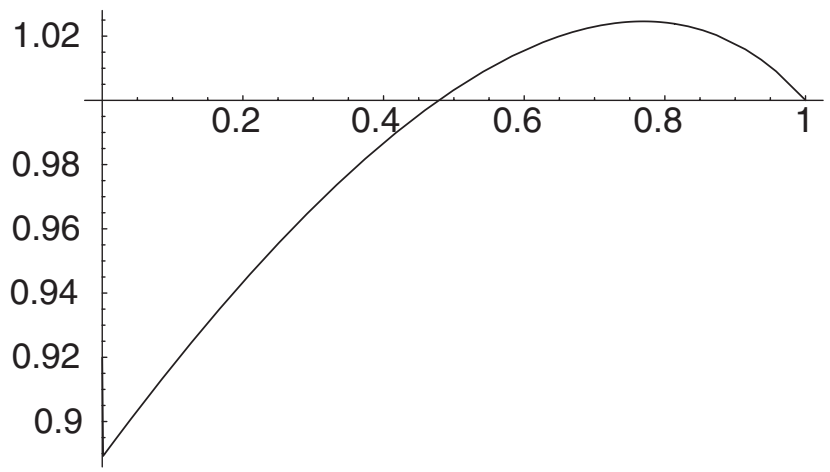

FIG. 2. The values of $f$ for $Y^{p, q}$. 


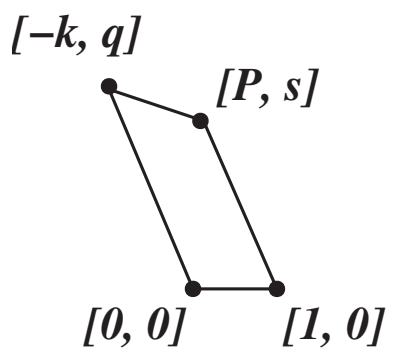

FIG. 3. Toric diagram of $L^{p, q, r}$. The integers $p, q, r, s$, and $P$ are taken such that $p+q=r+s, k s+q P=r, k>0$, and $0 \leq$ $p \leq r \leq s \leq q$.

$$
\frac{8}{9} \leq f(x, y) \leq 1.02459 .
$$

\section{B. Toric diagrams with five vertices}

\section{1. $X^{p, q}$}

$X^{p, q}$ is given by a specific toric diagram with five vertices (Fig. 4) [30]. The number of bosons are $\frac{N_{B}}{N^{2}}=6 p+$ $2 q+2$. We can compute the volume of $X^{p, q}$ using the $Z$-minimization procedure [10], but its analytic expression is difficult to find. Therefore we performed a numerical analysis and obtained the following range

$$
\frac{8}{9} \leq f \leq 1.03729 .
$$

The maximum is realized when $(p, q)=(4,3)$.

\section{Symmetric pentagon}

To find many more examples, we consider the five vertex toric diagram defined by Fig. 5. It has the reflection symmetry along the vertical axis. ${ }^{6}$ Note also that this toric diagram can be regarded as a deformation of that of the second delPezzo surface $d P_{2}$. The numbers of fields are $\frac{N_{B}}{N^{2}}=12 p r-4 p q$. Notice that since integer points are included on the edges of the toric diagram, the resulting manifold also includes orbifold singularities. ${ }^{7}$

We can perform the $Z$-minimization analytically in this case and the Reeb vector is found as $(3,0$, $\left.\frac{3\left(4 q r-r^{2}-\sqrt{8 q r^{3}+r^{4}}\right)}{4(q-r)}\right)$. Then $f$ is given in terms of $y \equiv \frac{q}{r}(0 \leq$ $y \leq 1$ ) by

${ }^{6}$ This symmetry allowed us to set the Reeb vector of the form $(3,0, z)$, which largely reduces the amount of the computations of $Z$-minimization.

${ }^{7}$ This means that these toric diagrams do not generically satisfy the "goodness" condition in [28]. However, the corresponding manifolds and their dual gauge theories are physically sensible as the orbifolds $T^{1,1} / Z_{p}$ are. Also if we would like to keep the diagrams good, we can consider limits of good toric diagrams. If we take the coordinates $\left(p_{i}, q_{i}\right)$ very large, we can approach the symmetric pentagon as much as we want because the function $f$ is invariant under the total scaling of the diagram.

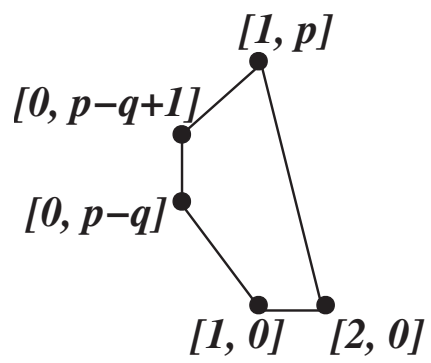

FIG. 4. Toric diagram of $X^{p, q}$.

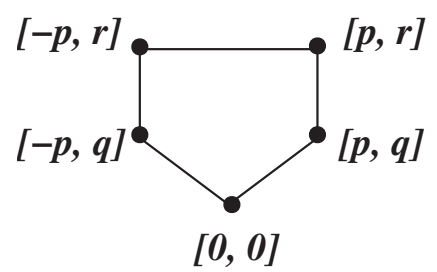

FIG. 5. Toric diagram of a symmetric pentagon.

$$
f(y)=\frac{32(3-y)(1-y)^{3}(1+\sqrt{8 y+1})}{27(3-\sqrt{8 y+1})(1+\sqrt{8 y+1}-4 y)^{2}} .
$$

This expression shows us the function $f$ takes the range

$$
\frac{8}{9} \leq f(y) \leq 1.03172 .
$$

Finally, we would like to summarize the central charge and the $R$-charges of baryons in this example as follows

$$
\begin{aligned}
a & =\frac{27 p\left(3 r^{2}-\sqrt{8 q r^{3}+r^{4}}\right)\left(r^{2}-4 q r+\sqrt{8 q r^{3}+r^{4}}\right)^{2}}{128(r-q)^{3}\left(r^{2}+\sqrt{8 q r^{3}+r^{4}}\right)} N^{2}, \\
R_{1} & =R_{4}=\frac{(1+\sqrt{8 y+1}-4 y)(3-\sqrt{8 y+1})}{4(1-y)^{2}(1+\sqrt{8 y+1})} N, \\
R_{2} & =R_{3}=\frac{(1+\sqrt{8 y+1}-4 y)^{2}}{4(1-y)^{2}(1+\sqrt{8 y+1})} N, \\
R_{5} & =\frac{2 y(3-\sqrt{8 y+1})}{(1-x)(1+\sqrt{8 y+1})} N .
\end{aligned}
$$

\section{Toric diagrams with six vertices}

\section{1. $Z^{p, q}$}

The Sasaki-Einstein manifold $Z^{p, q}$ is defined by the toric diagram with six vertices in Fig. 6 [31]. The number of bosons is $\frac{N_{B}}{N^{2}}=6 p+2 q+4$.

The volume of $Z^{p, q}$ is given as follows [31]

$$
\operatorname{Vol}\left(Z^{p, q}\right)=\pi^{3} \cdot \frac{9 p^{3}-9 p^{2} q+6 p q y_{2}-2 y_{2}^{2}}{3 y_{2}^{2}\left(3 p-y_{2}\right)^{2}} .
$$

Here $y_{2}$ is the solution to the cubic equation $2 y^{3}-$ $9 p q y^{2}-9 p^{2}(2 p-3 q) y+27 p^{3}(p-q)=0$ which lives in the region $0<y_{2}<3 p$. We find the function $f$ takes the 


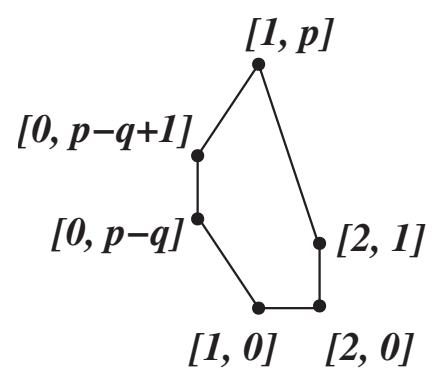

FIG. 6. Toric diagram of $Z^{p, q}$.

following values

$$
\frac{8}{9} \leq f \leq 1.05007
$$

The maximum value is again taken for $Z^{4,3}$.

\section{Symmetric hexagon}

As another example of toric diagrams with six vertices, ${ }^{8}$ we consider the ones with the reflection symmetries along the horizontal and vertical directions described by Fig. 7. This symmetry allows us to set the Reeb vector equal to $(3,0,0)$. The number of bosons is $\frac{N_{B}}{N^{2}}=12 q(p+r)$. In this case, the function $f$ does not depend on $q$ as in the case of the symmetric pentagon. Define $x \equiv \frac{r}{p}(0 \leq x \leq 1)$ and then the analytic expression of the function $f(x)$ is simply given by

$$
f(x)=\frac{4(2-x)(1+x)}{9} .
$$

Thus we obtain

$$
\frac{8}{9} \leq f(x) \leq 1
$$

The maximum value is taken when $x=1 / 2$, which corresponds to the third delPezzo surface $d P_{3}$. The minimum value is realized when $x=0,1$, i.e. orbifolds of $T^{1,1}$.

Note that the central charge and $R$ charges of baryons are given by the following expressions

$$
\begin{aligned}
a & =\frac{27 p^{2} q}{16(2 p-r)} N^{2} \\
R_{1} & =R_{2}=R_{4}=R_{5}=\frac{1}{2(2-x)} N \\
R_{3} & =R_{6}=\frac{1}{2-x} N
\end{aligned}
$$

\section{Toric diagram with infinitely many vertices}

It is very important to find how much the upper bound of the function $f$ increases as we raise the number $n$ of

\footnotetext{
${ }^{8}$ Notice that as in the symmetric pentagon, the corresponding manifolds will have orbifold singularities and the same footnote as in Sec. III B 2 applies.
}

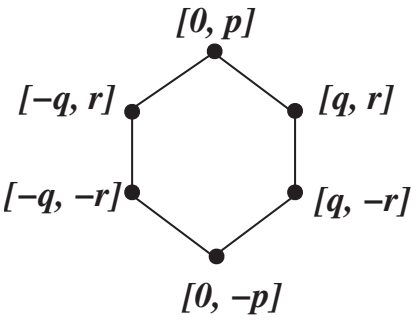

FIG. 7. Toric diagram of a symmetric hexagon.

vertices in toric diagrams. As the easiest example for general $n$ we concentrate on the specific example of the most symmetric toric diagram (i.e. the regular polygons) whose vertices are given by $\rho\left(\cos \left(\frac{2 \pi}{n} i\right), \sin \left(\frac{2 \pi}{n} i\right)\right),(i=$ $1,2, \ldots, n)$. The value of $f$ does not depend on $\rho$ because the function $f$ is invariant under the total rescaling of the toric diagram. Therefore, we can realize this diagram as a limit of very large toric diagrams taking $\rho \rightarrow \infty$. Then, because of the $Z_{2} \times Z_{2}$ symmetry, it is clear that the Reeb vector is given by $(3,0,0)$. Thus it is direct to compute its volume and the function $f$. The numbers of fields in the dual gauge theory are $\frac{N_{\text {gauge }}}{N^{2}}=n \sin \left(\frac{2 \pi}{n}\right)$ and $\frac{N_{\text {matter }}}{N^{2}}=$ $2 n \sin \left(\frac{2 \pi}{n}\right)$ for $n$ even, $\frac{N_{\text {matter }}}{N^{2}}=2 n \sin \left(\frac{\pi}{n}\right)\left(1+\cos \left(\frac{\pi}{n}\right)\right)$ for $n$ odd (we set $\rho=1$ ). The function $f$ becomes

$$
n: \text { even } \quad f(n)=\frac{n^{2}}{9} \sin ^{2}\left(\frac{\pi}{n}\right) \rightarrow \frac{\pi^{2}}{9} \quad(n \rightarrow \infty),
$$

$$
\begin{aligned}
n: \text { odd } \quad f(n) & =\frac{n^{2}}{27} \frac{\sin ^{2}\left(\frac{\pi}{n}\right)}{\cos \left(\frac{\pi}{n}\right)}\left(1+2 \cos \left(\frac{\pi}{n}\right)\right) \rightarrow \frac{\pi^{2}}{9} \\
(n \rightarrow \infty) . &
\end{aligned}
$$

Thus it does not become so large even if we increase the number of vertices. Its range is

$$
\frac{8}{9} \leq f(n)<\frac{\pi^{2}}{9}=1.09662
$$

The minimum value is attained when $n=4$, i.e. $T^{1,1}$.

We summarized all of the previous examples in this section in the Tables I and II. We also added a nontoric example $d P_{4}$ and compared it with the toric counterpart $\mathrm{PdP}_{4}$, whose central charges were computed in [32]. We also examined a new example whose toric diagram is described by a symmetric octagon.

\section{COMPARISON WITH $\mathcal{N}=1$ SQCD}

The function $f$ can be calculated only from the gauge theoretic data, i.e. the central charge and the number of bosons as is clear from (2.4). Therefore it will be useful to compare our previous analysis for the $\mathcal{N}=1$ quiver gauge theories which have AdS duals, with the one for the $\mathcal{N}=1$ SQCDs whose AdS duals have not been 
known. Since we are interested in SCFTs, we concentrate on the conformal window $\frac{3}{2} N_{c} \leq N_{f} \leq 3 N_{c}$ of the SQCD with the $S U\left(N_{c}\right)$ gauge group and $S U\left(N_{f}\right)$ flavor group. It is well known that at the IR fixed point $2 N_{f} N_{c}$ quarks $\psi_{q}$, $\psi_{\tilde{q}}$ and $N_{c}^{2}-1$ gauginos $\lambda$ have $R$ charge $R\left(\psi_{q}\right)=$ $R\left(\psi_{\tilde{q}}\right)=-\frac{N_{c}}{N_{f}}$ and $R(\lambda)=1$, respectively. Thus the central charge $[33,34]$ in the IR fixed point is given by

$$
a^{\mathrm{IR}}=\frac{3}{32}\left(3 \operatorname{Tr} R^{3}-\operatorname{Tr} R\right)=\frac{3}{16}\left(2 N_{c}^{2}-1-\frac{3 N_{c}^{4}}{N_{f}^{2}}\right) .
$$

Then we find the function $f$ is given by

$$
f=\frac{2 N_{f} N_{c}+N_{c}^{2}-1}{6 N_{c}^{2}-3-\frac{9 N_{c}^{4}}{N_{f}^{2}}} .
$$

In the planar limit $N_{c} \gg 1$ setting $x \equiv \frac{N_{f}}{N_{c}}$ finite, the function $f(x)$ is expressed by

$$
f(x)=\frac{x^{2}(1+2 x)}{6 x^{2}-9} .
$$

In the conformal window $\frac{3}{2} \leq x \leq 3$, this takes

$$
1.30248 \leq f(x) \leq 2 \text {. }
$$

The maximum and minimum values are taken for $x=3 / 2$ and $x=2.27163$, respectively.

\section{CONCLUSIONS AND DISCUSSIONS}

In this paper we have studied the ratio $\frac{S_{\text {free }}}{S_{\text {strong }}}=\frac{4}{3} f$ of the free Yang-Mills entropy to the entropy in the strongly coupled $\mathcal{N}=1$ SCFTs via the AdS/CFT correspondence. We mainly considered $\mathcal{N}=1$ SCFTs dual to toric SasakiEinstein manifolds $X_{5}$. Since they are classified by toric diagrams, we could compute the ratio rather systematically for infinitely many examples, though we could not exhaust all toric Sasaki-Einstein manifolds. We checked that in all examples the ratio takes the finite values within a rather narrow range (for a standard choice of the toric phase)

$$
\frac{8}{9} \leq f \leq f_{\max } .
$$

For example, the values of $f$ for $Y^{p, q}$ and $L^{p, q, r}$ are included in the range $\frac{8}{9} \leq f \leq 1.02459$. We can think remarkable even the fact that the ratio takes a finite value of order 1 . Even though the central charge $a$ is often used to characterize a given SCFT, it can take any arbitrary large values.

From the infinitely many examples which we explicitly examined in this paper, we can find the maximal value $f_{\text {our } \max }=1.09662$. This maximal value, however, seems to increase if we include other examples of toric diagrams which we did not consider in this paper. We would like to conjecture that the true upper bound $f_{\max }$ is only slightly larger than $f_{\text {our max }}$, say $f_{\text {max }} \sim 1.2$. We gave an evidence for this behavior by presenting an explicit analysis when the toric diagram is the regular $n$ polygon. On the other hand, the lowest bound $f=\frac{8}{9}$ is realized when $X_{5}$ is (an orbifold of ) $T^{1,1}$. Also notice that since $\frac{S_{\text {free }}}{S_{\text {strong }}}=\frac{4}{3} f$ is always greater than 1, the degrees of freedom in a strongly coupled Yang-Mills theory is smaller than those in the free Yang-Mills theory. This is natural since the interactions generally give masses to the off-diagonal elements of massless fields.

In most of the computations, we assumed a particular choice (3.1) of the toric phase. The ratio $f$ takes slightly different values when we employ different phases. We checked that this ambiguity does not spoil our semiquantitative argument in the explicit example of $Y^{p, q}$. To understand this issue in detail we need to develop a systematical way to analyze various toric phases and we left it as an important future problem.

Our result strongly suggests that the entropy ratios for all such $\mathcal{N}=1$ SCFTs deviate from the ones for the $\mathcal{N}=4$ super Yang-Mills theory (i.e. $f_{N=4}=1$ ) only by a small amount $(\sim \pm 20 \%)$. This means that the degrees of freedom of the strongly coupled SCFTs are not so different from the ones obtained in their free Yang-Mills counterparts. This may be a bit surprising since we know that such a $\mathcal{N}=1$ SCFT is realized as a nontrivial IR fixed point of an interacting $\mathcal{N}=1$ quiver gauge theory [5]. Indeed, we have seen that in the conformal window of $\mathcal{N}=1 \mathrm{SQCD}$, $f$ takes slightly larger values than the range (5.1).

We would like to mention a possibility that our result (5.1) may be a special property which is common to all $\mathcal{N}=1$ SCFTs with AdS duals. Our analysis of the ratio $f$ may be regarded as a first step to explore an index which gives the criterion of the existence of AdS dual. Thus an interesting future problem is to compute $f_{\max }$ for all toric diagrams, and also to see if the situation does not change when we extend the examples to nontoric ones.

We would also like to stress that the ratio $f$ is mathematically well defined for all toric Sasaki-Einstein manifolds in that it takes a unique value corresponding to a toric diagram. Thus, even the fact that the ratio $f$ is always finite for any toric Sasaki-Einstein manifold is already a nontrivial mathematical result.

Finally we would like to point out that we can define other ratios which are computable from the central charges and field contents of $\mathcal{N}=1$ SCFTs. One such example is the ratio $g=\frac{N_{\text {gauge }}}{4 a}$ using the number $N_{\text {gauge }}$ of vector multiplets, instead of our previous ratio $f=\frac{N_{B}}{16 a}$. We can again show $g=1$ for orbifold quiver gauge theories. For example, in the $\mathcal{N}=1$ SCFTs dual to $Y^{p, q}$ this new ratio $g$ takes the values within the range $1 \leq g \leq \frac{32}{27}$, where the maximum is taken when $X_{5}$ is (an orbifold of) $T^{1,1}$. We generally expect the range $1 \leq g \leq g_{\max }\left(g_{\max } \sim O(1)\right)$ for all $\mathcal{N}=1$ SCFTs dual to toric Sasaki-Einstein manifolds.

Two more interesting quantities will be $h_{a}=\frac{a_{\text {free }}}{a_{\mathrm{SCF}}}$ and $h_{c}=\frac{c_{\text {free }}}{c_{\text {SCFT }}}$, which are the ratios of the central charges $a$ and 
TATSUMA NISHIOKA AND TADASHI TAKAYANAGI

$c$ of the free Yang-Mills theory to the ones for the interacting SCFT. It is trivial to see that $h_{a}=h_{c}=1$ for any orbifold quiver gauge theories. For $Y^{p, q}$ we find the values $1 \leq h_{a} \leq 1.10352$ and $\frac{80}{81} \leq h_{c} \leq 1.05321$. Notice that $h_{a}$ is always greater than 1 while $h_{c}$ is not in this example. This suggests that there exist RG flows ${ }^{9}$ from orbifold theories to the interacting SCFTs since the central charge $a$ always decreases under the RG flow, while $c$ does not.

\footnotetext{
${ }^{9}$ We would like to thank Igor Klebanov very much for pointing out this possibility to us.
}

PHYSICAL REVIEW D 76, 044004 (2007)

\section{ACKNOWLEDGMENTS}

T. T. would like to thank H. Isono, I. Klebanov, T. Oota, Y. Tachikawa, M. Yamazaki, and Y. Yasui for providing us important comments and references. T. N. is grateful to the members of the Theoretical Particle Physics Group in Kyoto University for useful discussions and encouragements during the preparations of his master thesis, part of which is based on the present paper. The work of T. T. is supported in part by JSPS Grant-in-Aid for Scientific Research No. 18840027.
[1] J. M. Maldacena, Adv. Theor. Math. Phys. 2, 231 (1998); Int. J. Theor. Phys. 38, 1113 (1999); O. Aharony, S. S. Gubser, J. M. Maldacena, H. Ooguri, and Y. Oz, Phys. Rep. 323, 183 (2000).

[2] J.P. Gauntlett, D. Martelli, J. Sparks, and D. Waldram, Adv. Theor. Math. Phys. 8, 711 (2004).

[3] S. Benvenuti, S. Franco, A. Hanany, D. Martelli, and J. Sparks, J. High Energy Phys. 06 (2005) 064.

[4] M. Cvetic, H. Lu, D. N. Page, and C. N. Pope, Phys. Rev. Lett. 95, 071101 (2005).

[5] I. R. Klebanov and E. Witten, Nucl. Phys. B536, 199 (1998).

[6] S. Benvenuti, B. Feng, A. Hanany, and Y.H. He, arXiv:hep-th/0608050; D. Martelli and J. Sparks, Nucl. Phys. B759, 292 (2006); A. Basu and G. Mandal, arXiv:hep-th/0608093.

[7] S. S. Gubser, I. R. Klebanov, and A. W. Peet, Phys. Rev. D 54, 3915 (1996).

[8] E. Witten, Adv. Theor. Math. Phys. 2, 505 (1998).

[9] O. Aharony, J. Marsano, S. Minwalla, K. Papadodimas, and M. Van Raamsdonk, Adv. Theor. Math. Phys. 8, 603 (2004); B. Sundborg, Nucl. Phys. B573, 349 (2000).

[10] D. Martelli, J. Sparks, and S. T. Yau, Commun. Math. Phys. 268, 39 (2006); arXiv:hep-th/0603021.

[11] K. Intriligator and B. Wecht, Nucl. Phys. B667, 183 (2003).

[12] A. Butti and A. Zaffaroni, J. High Energy Phys. 11 (2005) 019; Fortschr. Phys. 54, 309 (2006).

[13] Y. Tachikawa, Nucl. Phys. B733, 188 (2006).

[14] E. Barnes, E. Gorbatov, K. Intriligator, and J. Wright, Nucl. Phys. B732, 89 (2006).

[15] S. Franco, A. Hanany, K. D. Kennaway, D. Vegh, and B. Wecht, J. High Energy Phys. 01 (2006) 096.

[16] S. Franco, A. Hanany, D. Martelli, J. Sparks, D. Vegh, and
B. Wecht, J. High Energy Phys. 01 (2006) 128.

[17] A. Hanany and D. Vegh, arXiv:hep-th/0511063.

[18] B. Feng, Y.H. He, K.D. Kennaway, and C. Vafa, arXiv:hep-th/0511287.

[19] Y. Imamura, J. High Energy Phys. 06 (2006) 011; 12 (2006) 041.

[20] Y. Imamura, H. Isono, K. Kimura, and M. Yamazaki, arXiv:hep-th/0702049.

[21] S. S. Gubser, Phys. Rev. D 59, 025006 (1998).

[22] J. L. Cardy, Phys. Lett. B 215, 749 (1988).

[23] S. Benvenuti, A. Hanany, and P. Kazakopoulos, J. High Energy Phys. 07 (2005) 021.

[24] G. T. Horowitz and R. C. Myers, Phys. Rev. D 59, 026005 (1998).

[25] S. Ryu and T. Takayanagi, J. High Energy Phys. 08 (2006) 045; Phys. Rev. Lett. 96, 181602 (2006).

[26] T. Nishioka and T. Takayanagi, J. High Energy Phys. 01 (2007) 090

[27] A. Kato, arXiv:hep-th/0610266.

[28] A. Futaki, H. Ono, and G. Wang, arXiv:math.dg/0607586; K. Cho, A. Futaki, and H. Ono, arXiv:math.dg/0701122.

[29] S. Benvenuti and M. Kruczenski, J. High Energy Phys. 04 (2006) 033; A. Butti, D. Forcella, and A. Zaffaroni, J. High Energy Phys. 09 (2005) 018.

[30] A. Hanany, P. Kazakopoulos, and B. Wecht, J. High Energy Phys. 08 (2005) 054.

[31] T. Oota and Y. Yasui, Nucl. Phys. B762, 377 (2007).

[32] A. Butti, D. Forcella, and A. Zaffaroni, J. High Energy Phys. 02 (2007) 081.

[33] D. Anselmi, D. Z. Freedman, M. T. Grisaru, and A. A. Johansen, Nucl. Phys. B526, 543 (1998).

[34] D. Anselmi, J. Erlich, D.Z. Freedman, and A. A. Johansen, Phys. Rev. D 57, 7570 (1998). 